\title{
Global Strong Solution to the Density-Dependent 2-D Liquid Crystal Flows
}

\author{
Yong Zhou, ${ }^{1}$ Jishan Fan, ${ }^{2}$ and Gen Nakamura ${ }^{3}$ \\ ${ }^{1}$ Department of Mathematics, Zhejiang Normal University, Jinhua 321004, China \\ ${ }^{2}$ Department of Applied Mathematics, Nanjing Forestry University, Nanjing 210037, China \\ ${ }^{3}$ Department of Mathematics, Inha University, Incheon 402-751, Republic of Korea
}

Correspondence should be addressed to Yong Zhou; yzhoumath@zjnu.edu.cn

Received 6 November 2012; Accepted 14 February 2013

Academic Editor: Giovanni P. Galdi

Copyright (c) 2013 Yong Zhou et al. This is an open access article distributed under the Creative Commons Attribution License, which permits unrestricted use, distribution, and reproduction in any medium, provided the original work is properly cited.

\begin{abstract}
The initial-boundary value problem for the density-dependent flow of nematic crystals is studied in a 2-D bounded smooth domain. For the initial density away from vacuum, the existence and uniqueness is proved for the global strong solution with the large initial velocity $u_{0}$ and small $\nabla d_{0}$. We also give a regularity criterion $\nabla d \in L^{p}\left(0, T ; L^{q}(\Omega)\right)((2 / q)+(2 / p)=1,2<q \leq \infty)$ of the problem with the Dirichlet boundary condition $u=0, d=d_{0}$ on $\partial \Omega$.
\end{abstract}

\section{Introduction and Main Results}

Let $\Omega \subseteq \mathbb{R}^{2}$ be a bounded domain with smooth boundary $\partial \Omega$, and $v$ is the unit outward normal vector on $\partial \Omega$. We consider the global strong solution to the density-dependent incompressible liquid crystal flow [1-4] as follows:

$$
\begin{gathered}
\operatorname{div} u=0, \\
\partial_{t} \rho+\operatorname{div}(\rho u)=0, \\
\partial_{t}(\rho u)+\operatorname{div}(\rho u \otimes u)+\nabla \pi-\Delta u=-\nabla \cdot(\nabla d \odot \nabla d), \\
\partial_{t} d+u \cdot \nabla d-\Delta d=|\nabla d|^{2} d,
\end{gathered}
$$

in $(0, \infty) \times \Omega$ with initial and boundary conditions

$$
\begin{gathered}
(\rho, u, d)(\cdot, 0)=\left(\rho_{0}, u_{0}, d_{0}\right) \quad \text { in } \Omega, \\
u=0, \quad \partial_{v} d=0 \quad \text { on } \partial \Omega,
\end{gathered}
$$

where $\rho$ denotes the density, $u$ the velocity, $d$ the unit vector field that represents the macroscopic molecular orientations, and $\pi$ the pressure. The symbol $\nabla d \odot \nabla d$ denotes a matrix whose $(i, j)$ th entry is $\partial_{i} d \partial_{j} d$, and it is easy to find that $\nabla d \odot$ $\nabla d=\nabla d^{T} \nabla d$.
When $d$ is a given constant unit vector, then (1), (2), and (3) represent the well-known density-dependent NavierStokes system, which has received many studies; see [5-7] and references therein.

When $\rho \equiv 1$ and $\Omega:=\mathbb{R}^{2}$, Xu and Zhang [8] proved global existence of weak solutions to the problem if $u_{0} \in$ $L^{2}, \nabla d_{0} \in L^{2},\left|d_{0}\right|=1$, and

$$
\exp \left(216\left(\left\|u_{0}\right\|_{L^{2}}^{2}+\frac{1}{16}\right)^{2}\right)\left\|\nabla d_{0}\right\|_{L^{2}}^{2}<\frac{1}{16} .
$$

When $\rho \equiv 1$ and (6) is replaced by

$$
u=0, \quad d=d_{0} \quad \text { on } \partial \Omega .
$$

Lin et al. [9] proved the global existence of weak solutions to the system (1)-(5) and (8), which are smooth away from at most finitely many singular times, and they also prove a regularity criterion

$$
d \in L^{2}\left(0, T ; H^{2}(\Omega)\right) .
$$

When $\rho=1$ and the term $|\nabla d|^{2}$ in (4) is replaced by (1 $\left.|d|^{2}\right) d$, then the problem has been studied in [10-15].

Very recently, Wen and Ding [16] proved the global existence and uniqueness of strong solutions to the problem (1)(6) with small $u_{0}$ and $\nabla d_{0}$ and the local strong solutions with large initial data when $\Omega \subseteq \mathbb{R}^{2}$ is a smooth bounded domain. 
Fan et al. [17] studied the regularity criterion of the Cauchy problem (1)-(5) when $\Omega:=\mathbb{R}^{2}$.

We will prove the following.

Theorem 1. Let $0<m \leq \rho_{0} \leq M<\infty, \rho_{0} \in W^{1, r}$ for some $r \in(2, \infty), u_{0} \in H_{0}^{1} \cap H^{2}$, and $d_{0} \in H^{3}$ with $\operatorname{div} u_{0}=0$, and $\left|d_{0}\right|=1$ in $\Omega$. If

$$
\left\|\nabla d_{0}\right\|_{L^{2}}^{2} \exp \left[216 \frac{C_{0}^{2}}{m}\left(\left\|\sqrt{\rho_{0}} u_{0}\right\|_{L^{2}}^{2}+\frac{1}{8 C_{0}^{2}}\right)^{2}\right] \leq \frac{1}{8 C_{0}^{2}},
$$

with an absolute constant $C_{0}$ in (22), then the problem (1)-(6) has a unique global-in-time strong solution $(\rho, u, d)$ satisfying

$$
\begin{gathered}
\|\rho\|_{L^{\infty}\left(0, T ; W^{1, r}\right)} \leq C, \quad\left\|\rho_{t}\right\|_{L^{\infty}\left(0, T ; L^{r}\right)} \leq C, \\
\|u\|_{L^{\infty}\left(0, T ; H^{2}\right) \cap L^{2}\left(0, T ; W^{2, s}\right)} \leq C, \quad \text { forsome } s>2,
\end{gathered}
$$

$$
\|d\|_{L^{\infty}\left(0, T ; H^{3}\right)} \leq C .
$$

Remark 2. When $\Omega:=\mathbb{R}^{2}$, Theorem 1 is also correct, thus improving the result in [18], where $u_{0}$ and $\nabla d_{0}$ are assumed to be small.

Next, we consider (1)-(4) with $\rho \equiv 1$ as follows:

$$
\begin{gathered}
\operatorname{div} u=0, \\
\partial_{t} u+u \cdot \nabla u+\nabla \pi-\Delta u=-\nabla \cdot(\nabla d \odot \nabla d), \\
\partial_{t} d+u \cdot \nabla d-\Delta d=|\nabla d|^{2} d, \\
u=0, \quad d=d_{0} \quad \text { on } \partial \Omega, \\
(u, d)(\cdot, 0)=\left(u_{0}, d_{0}\right) \quad \text { in } \Omega .
\end{gathered}
$$

We will prove the following.

Theorem 3. Let $u_{0} \in L^{2}$ and $d_{0} \in H^{1}$ with $\operatorname{div} u_{0}=0$ and $\left|d_{0}\right|=1$ in $\Omega$ and $d_{0} \in C^{2, \beta}(\partial \Omega)$ for some $\beta \in(0,1)$. If $d$ satisfies

$$
\nabla d \in L^{q}\left(0, T ; L^{p}\right), \quad \frac{2}{q}+\frac{2}{p}=1, \quad 2<p \leq \infty,
$$

then the strong solution $(u, d)$ can be extended beyond $T>0$.

Remark 4. In [9], the authors prove the regularity criterion (9) for the problem (12)-(16), and our condition (17) is weaker than (9). Moreover, (17) is scaling invariant for (12)-(14).

\section{Proof of Theorem 1}

This section is devoted to the proof of Theorem 1 . Since the local-in-time well-posedness has been proved in [16], we only need to establish a priori estimates. Also, by the local well-posedness result in [16], we note that $\nabla d$ is absolutely continuous on $[0, T]$ for any given $T>0$.

By the maximum principle, it follows from (1) and (2) that

$$
0<m \leq \rho \leq M<\infty .
$$

Testing (3) by $u$ and using (1) and (2), we see that

$$
\frac{1}{2} \frac{d}{d t} \int \rho u^{2} d x+\int|\nabla u|^{2} d x=-\int(u \cdot \nabla) d \cdot \Delta d d x .
$$

Testing (4) by $-\Delta d-|\nabla d|^{2} d$, using $|d|=1$, we find that

$$
\frac{1}{2} \frac{d}{d t} \int|\nabla d|^{2} d x+\left.\left.\int|\Delta d+| \nabla d\right|^{2} d\right|^{2} d x=\int(u \cdot \nabla) d \cdot \Delta d d x
$$

Summing up (19) and (20) and integrating over $(0, T)$, we get

$$
\begin{gathered}
\int\left(\rho u^{2}+|\nabla d|^{2}\right) d x+2 \int_{0}^{T} \int\left(|\nabla u|^{2}+\left.|\Delta d+| \nabla d\right|^{2} d \mid\right) d x d t \\
\leq \int\left(\rho_{0} u_{0}^{2}+\left|\nabla d_{0}\right|^{2}\right) d x .
\end{gathered}
$$

Since $\partial_{v} d=0$ on $(0, \infty) \times \partial \Omega$, we have the following Gagliardo-Nirenberg inequality:

$$
\|\nabla d\|_{L^{4}}^{2} \leq C_{0}\|\nabla d\|_{L^{2}}\|\Delta d\|_{L^{2}}
$$
derive

By (20) and the Ladyzhenskaya inequality in 2D, we

$\frac{1}{2} \frac{d}{d t} \int|\nabla d|^{2} d x+\left.\left.\int|\Delta d+| \nabla d\right|^{2} d\right|^{2} d x$

$\leq\|u\|_{L^{4}}\|\nabla d\|_{L^{4}}\|\Delta d\|_{L^{2}}$

$$
\begin{aligned}
& \leq \sqrt{2}\|u\|_{L^{2}}^{1 / 2}\|\nabla u\|_{L^{2}}^{1 / 2} \cdot \sqrt{C_{0}}\|\nabla d\|_{L^{2}}^{1 / 2}\|\Delta d\|_{L^{2}}^{3 / 2} \\
& \leq \frac{\|\Delta d\|_{L^{2}}^{2}}{8}+216 C_{0}^{2}\|u\|_{L^{2}}^{2}\|\nabla u\|_{L^{2}}^{2}\|\nabla d\|_{L^{2}}^{2} \\
& \leq \frac{\|\Delta d\|_{L^{2}}^{2}}{8}+216 \frac{C_{0}^{2}}{m}\left(\left\|\sqrt{\rho_{0}} u_{0}\right\|_{L^{2}}^{2}+\left\|\nabla d_{0}\right\|_{L^{2}}^{2}\right)\|\nabla u\|_{L^{2}}^{2}\|\nabla d\|_{L^{2}}^{2} .
\end{aligned}
$$

On the other hand, since $(a+b)^{2} \geq\left(a^{2} / 2\right)-b^{2}$, we have

$$
\begin{gathered}
\left.\left.\int|\Delta d+| \nabla d\right|^{2} d\right|^{2} d x \geq \frac{\|\Delta d\|_{L^{2}}^{2}}{2}-\|\nabla d\|_{L^{4}}^{4} \\
\geq \frac{\|\Delta d\|_{L^{2}}^{2}}{2}-C_{0}^{2}\|\nabla d\|_{L^{2}}^{2}\|\Delta d\|_{L^{2}}^{2} .
\end{gathered}
$$

If the initial data $\left\|\nabla d_{0}\right\|_{L^{2}}^{2}<\left(1 / C_{0}^{2}\right)(1 / 8)$, then there exists $T_{1}>0$ such that for any $t \in\left[0, T_{1}\right]$,

$$
\|\nabla d(t)\|_{L^{2}}^{2} \leq \frac{1}{C_{0}^{2}} \cdot \frac{1}{4}
$$


We denote by $T_{1}^{*}$ the maximal time such that (25) holds on $\left[0, T_{1}^{*}\right]$. Therefore, by (23), (24), and (25), it follows that for any $t \in\left[0, T_{1}^{*}\right]$,

$$
\begin{aligned}
& \frac{d}{d t} \int|\nabla d|^{2} d x+\frac{1}{4}\|\Delta d\|_{L^{2}}^{2} \\
& \quad \leq 432 \frac{C_{0}^{2}}{m}\left(\left\|\sqrt{\rho_{0}} u_{0}\right\|_{L^{2}}^{2}+\left\|\nabla d_{0}\right\|_{L^{2}}^{2}\right)\|\nabla u\|_{L^{2}}^{2}\|\nabla d\|_{L^{2}}^{2} \\
& \quad \leq 432 \frac{C_{0}^{2}}{m}\left(\left\|\sqrt{\rho_{0}} u_{0}\right\|_{L^{2}}^{2}+\frac{1}{8 C_{0}^{2}}\right)\|\nabla u\|_{L^{2}}^{2}\|\nabla d\|_{L^{2}}^{2},
\end{aligned}
$$

which gives

$$
\begin{aligned}
& \|\nabla d(t)\|_{L^{2}}^{2}+\frac{1}{4} \int_{0}^{t}\|\Delta d(\tau)\|_{L^{2}}^{2} d \tau \\
& \leq\left\|\nabla d_{0}\right\|_{L^{2}}^{2} \exp \left[432 \frac{C_{0}^{2}}{m}\left(\left\|\sqrt{\rho_{0}} u_{0}\right\|_{L^{2}}^{2}+\frac{1}{8 C_{0}^{2}}\right)\right. \\
& \left.\quad \times \int_{0}^{T_{1}^{*}}\|\nabla u\|_{L^{2}}^{2} d \tau\right] \\
& \leq\left\|\nabla d_{0}\right\|_{L^{2}}^{2} \exp \left[216 \frac{C_{0}^{2}}{m}\left(\left\|\sqrt{\rho_{0}} u_{0}\right\|_{L^{2}}^{2}+\frac{1}{8 C_{0}^{2}}\right)^{2}\right] \\
& \leq \frac{1}{8 C_{0}^{2}},
\end{aligned}
$$

which implies that $T_{1}^{*}=T$ if the initial data satisfies

$$
\left\|\nabla d_{0}\right\|_{L^{2}}^{2} \exp \left[216 \frac{C_{0}^{2}}{m}\left(\left\|\sqrt{\rho_{0}} u_{0}\right\|_{L^{2}}^{2}+\frac{1}{8 C_{0}^{2}}\right)^{2}\right] \leq \frac{1}{8 C_{0}^{2}} \text {. }
$$

Let $T^{*}$ be a maximal existence time for the solution $(\rho, u, d)$. Then, (18), (21), and (27) ensure that $T^{*}=\infty$ by continuity argument.

Testing (3) by $u_{t}$, using (1), (18), (21), (22), $|d|=1$, and the Gagliardo-Nirenberg inequalities, we obtain

$$
\begin{gathered}
\frac{1}{2} \frac{d}{d t} \int|\nabla u|^{2} d x+\int \rho u_{t}^{2} d x \\
=-\int \rho u \cdot \nabla u \cdot u_{t} d x-\int u_{t} \cdot \nabla d \cdot \Delta d d x \\
\leq C\left\|\sqrt{\rho} u_{t}\right\|_{L^{2}}\left(\|u\|_{L^{4}}\|\nabla u\|_{L^{4}}+\|\nabla d\|_{L^{4}}\|\Delta d\|_{L^{4}}\right) \\
\leq C\left\|\sqrt{\rho} u_{t}\right\|_{L^{2}}\left[\|u\|_{L^{2}}^{1 / 2}\|\nabla u\|_{L^{2}}\left(\|\Delta u\|_{L^{2}}^{1 / 2}+\|u\|_{L^{2}}^{1 / 2}\right)\right. \\
\left.+\|\nabla d\|_{L^{2}}^{1 / 2}\|\Delta d\|_{L^{2}}\left(\|\nabla \Delta d\|_{L^{2}}^{1 / 2}+\|d\|_{L^{2}}^{1 / 2}\right)\right] \\
\leq C\left\|\sqrt{\rho} u_{t}\right\|_{L^{2}}\left(\|\nabla u\|_{L^{2}}\|\Delta u\|_{L^{2}}^{1 / 2}+\|\nabla u\|_{L^{2}}+\|\Delta d\|_{L^{2}}\right. \\
\left.\times\|\nabla \Delta d\|_{L^{2}}^{1 / 2}+\|\Delta d\|_{L^{2}}\right) .
\end{gathered}
$$

On the other hand, (3) can be rewritten as

$-\Delta u+\nabla \pi=f:=-\rho u_{t}-\rho u \cdot \nabla u-\nabla \cdot(\nabla d \odot \nabla d)$.
By the $H^{2}$-theory of Stokes system, we have

$$
\begin{aligned}
& \|\Delta u\|_{L^{2}} \leq C\|f\|_{L^{2}} \\
& \leq C\left\|\sqrt{\rho} u_{t}\right\|_{L^{2}}+C\|u\|_{L^{4}}\|\nabla u\|_{L^{4}}+C\|\nabla d\|_{L^{4}}\|\Delta d\|_{L^{4}} \\
& \leq C\left\|\sqrt{\rho} u_{t}\right\|_{L^{2}}+C\|\nabla u\|_{L^{2}}\|\Delta u\|_{L^{2}}^{1 / 2}+C\|\nabla u\|_{L^{2}} \\
& \quad+C\|\Delta d\|_{L^{2}}\|\nabla \Delta d\|_{L^{2}}^{1 / 2}+C\|\Delta d\|_{L^{2}},
\end{aligned}
$$

which yields

$$
\begin{aligned}
\|\Delta u\|_{L^{2}} \leq & C\left\|\sqrt{\rho} u_{t}\right\|_{L^{2}}+C\|\nabla u\|_{L^{2}}^{2}+C \\
& +C\|\Delta d\|_{L^{2}}\|\nabla \Delta d\|_{L^{2}}^{1 / 2}+C\|\Delta d\|_{L^{2}} .
\end{aligned}
$$

Inserting (32) into (29), we deduce that

$$
\begin{aligned}
& \frac{1}{2} \frac{d}{d t} \int|\nabla u|^{2} d x+\int \rho u_{t}^{2} d x \\
& \leq C\left\|\sqrt{\rho} u_{t}\right\|_{L^{2}}^{3 / 2}\|\nabla u\|_{L^{2}}+C\left\|\sqrt{\rho} u_{t}\right\|_{L^{2}}\left(\|\nabla u\|_{L^{2}}^{2}+\|\nabla u\|_{L^{2}}\right) \\
& \quad+C\left\|\sqrt{\rho} u_{t}\right\|_{L^{2}}\|\Delta d\|_{L^{2}}\|\nabla \Delta d\|_{L^{2}}^{1 / 2}+C\left\|\sqrt{\rho} u_{t}\right\|_{L^{2}}\|\Delta d\|_{L^{2}} \\
& \leq \frac{1}{8}\left\|\sqrt{\rho} u_{t}\right\|_{L^{2}}^{2}+C\|\nabla u\|_{L^{2}}^{4}+C+\frac{1}{8}\|\nabla \Delta d\|_{L^{2}}^{2}+C\|\Delta d\|_{L^{2}}^{4} .
\end{aligned}
$$

Applying $\Delta$ to (4), testing by $\Delta d$, using $|d|=1,(21)$ and (22), and the Gagliardo-Nirenberg inequalities, we have

$$
\begin{aligned}
\frac{1}{2} \frac{d}{d t} \int|\Delta d|^{2} d x+\int|\nabla \Delta d|^{2} d x \\
\leq \int\left|\nabla\left(|\nabla d|^{2} d\right)\right||\nabla \Delta d| d x+\int|\nabla(u \cdot \nabla d)||\nabla \Delta d| d x \\
\leq C\left(\|\nabla d\|_{L^{6}}^{3}+\|\nabla d\|_{L^{4}}\|\Delta d\|_{L^{4}}+\|u\|_{L^{4}}\|\Delta d\|_{L^{4}}\right. \\
\left.\quad+\|\nabla u\|_{L^{2}}\|\nabla d\|_{L^{\infty}}\right)\|\nabla \Delta d\|_{L^{2}} \\
\leq C\left(\|\nabla d\|_{L^{2}}\|\Delta d\|_{L^{2}}^{2}+\|\Delta d\|_{L^{2}}\|\nabla \Delta d\|_{L^{2}}^{1 / 2}+\|\Delta d\|_{L^{2}}\right. \\
\quad+\|\nabla u\|_{L^{2}}^{1 / 2}\|\Delta d\|_{L^{2}}^{1 / 2}\|\nabla \Delta d\|_{L^{2}}^{1 / 2} \\
\quad+\|\nabla u\|_{L^{2}}^{1 / 2}\|\Delta d\|_{L^{2}}^{1 / 2}+\|\nabla u\|_{L^{2}} \\
\left.\quad \times\|\nabla d\|_{L^{2}}^{1 / 2}\|\nabla \Delta d\|_{L^{2}}^{1 / 2}\right)\|\nabla \Delta d\|_{L^{2}} \\
\leq \frac{1}{8}\|\nabla \Delta d\|_{L^{2}}^{2}+C\|\Delta d\|_{L^{2}}^{4}+C+C\|\nabla u\|_{L^{2}}^{4} .
\end{aligned}
$$

Here, we have used the Gagliardo-Nirenberg inequalities

$$
\begin{gathered}
\|\nabla d\|_{L^{6}}^{3} \leq C\|\nabla d\|_{L^{2}}\|\Delta d\|_{L^{2}}^{2}, \\
\|\nabla d\|_{L^{\infty}}^{2} \leq\|\nabla d\|_{L^{2}}\|\nabla \Delta d\|_{L^{2}}, \\
\|\Delta d\|_{L^{4}}^{2} \leq C\|\Delta d\|_{L^{2}}\|\nabla \Delta d\|_{L^{2}}+C\|\Delta d\|_{L^{2}} .
\end{gathered}
$$


Combining (33) and (34) and using the Gronwall inequality, we have

$$
\begin{gathered}
\|u\|_{L^{\infty}\left(0, T ; H^{1}\right)}+\|u\|_{L^{2}\left(0, T ; H^{2}\right)} \leq C, \\
\left\|\sqrt{\rho} u_{t}\right\|_{L^{2}\left(0, T ; L^{2}\right)} \leq C, \\
\|d\|_{L^{\infty}\left(0, T ; H^{2}\right)}+\|d\|_{L^{2}\left(0, T ; H^{3}\right)} \leq C .
\end{gathered}
$$

Now, by the similar calculations as those in [17], we arrive at

$$
\begin{gathered}
\left\|\left(u_{t}, \nabla d_{t}\right)\right\|_{L^{\infty}\left(0, T ; L^{2}\right) \cap L^{2}\left(0, T ; H^{1}\right)} \leq C, \\
\|(u, \nabla d)\|_{L^{\infty}\left(0, T ; H^{2}\right)} \leq C, \\
\|u\|_{L^{2}\left(0, T ; W^{2, s}\right)} \leq C \quad \text { for some } s>2, \\
\|\rho\|_{L^{\infty}\left(0, T ; W^{1, r}\right)} \leq C, \quad\left\|\rho_{t}\right\|_{L^{\infty}\left(0, T ; L^{r}\right)} \leq C .
\end{gathered}
$$

This completes the proof.

\section{Proof of Theorem 3}

This section is devoted to the proof of Theorem 3. By the results in [9], we only need to prove (9).

Similar to (21), we still have

$$
\begin{aligned}
& \int\left(u^{2}+|\nabla d|^{2}\right) d x+2 \int_{0}^{T} \int\left(|\nabla u|^{2}+\left.|\Delta d+| \nabla d\right|^{2} d \mid\right) d x d t \\
& \leq \int\left(u_{0}^{2}+\left|\nabla d_{0}\right|^{2}\right) d x .
\end{aligned}
$$
ties:

We will use the following Gagliardo-Nirenberg inequali-

$$
\begin{gathered}
\|u\|_{L^{2 p /(p-2)}} \leq C\|u\|_{L^{2}}^{1-(2 / p)}\|\nabla u\|_{L^{2}}^{2 / p}, \\
\|\nabla d\|_{L^{2 p /(p-2)}} \leq C\|\nabla d\|_{L^{2}}^{1-(2 / p)}\|\Delta d\|_{L^{2}}^{2 / p}+C\|\nabla d\|_{L^{2}} .
\end{gathered}
$$

Testing (14) by $-\Delta d$, using $|d|=1$, (40), (41), and (42), we have

$$
\begin{aligned}
& \frac{1}{2} \frac{d}{d t} \int|\nabla d|^{2} d x+\int|\Delta d|^{2} d x \\
& \quad=\int\left(u \cdot \nabla d-|\nabla d|^{2} d\right) \Delta d d x \\
& \leq\left(\|u\|_{L^{2 p /(p-2)}}\|\nabla d\|_{L^{p}}+\|\nabla d\|_{L^{p}}\|\nabla d\|_{L^{2 p /(p-2)}}\right)\|\Delta d\|_{L^{2}} \\
& \leq C\|\nabla d\|_{L^{p}}\left(\|u\|_{L^{2}}^{1-(2 / p)}\|\nabla u\|_{L^{2}}^{2 / p}+\|\nabla d\|_{L^{2}}\right. \\
& \left.\quad+\|\nabla d\|_{L^{2}}^{1-(2 / p)}\|\Delta d\|_{L^{2}}^{2 / p}\right)\|\Delta d\|_{L^{2}} \\
& \leq C\|\nabla d\|_{L^{p}}\left(\|\nabla u\|_{L^{2}}^{2 / p}+1+\|\Delta d\|_{L^{2}}^{2 / p}\right)\|\Delta d\|_{L^{2}}^{2} \\
& \leq \frac{1}{4}\|\Delta d\|_{L^{2}}^{2}+C\|\nabla d\|_{L^{p}}^{2}\left(\|\nabla u\|_{L^{2}}^{4 / p}+1+\|\Delta d\|_{L^{2}}^{4 / p}\right) \\
& \leq \frac{1}{2}\|\Delta d\|_{L^{2}}^{2}+\|\nabla u\|_{L^{2}}^{2}+C\|\nabla d\|_{L^{p}}^{2 p /(p-2)}+C,
\end{aligned}
$$

which gives (9).

This completes the proof.

\section{Acknowledgments}

The authors would like to thank the referees for careful reading and helpful suggestions. This work is partially supported by the Zhejiang Innovation Project (Grant no. T200905), the ZJNSF (Grant no. R6090109), and the NSFC (Grant no. 11171154).

\section{References}

[1] S. Chandrasekhar, Liquid Crystalsed, Cambridge University Press, 2nd edition, 1992.

[2] J. L. Ericksen, "Hydrostatic theory of liquid crystals," Archive for Rational Mechanics and Analysis, vol. 9, pp. 371-378, 1962.

[3] F. M. Leslie, "Some constitutive equations for liquid crystals," Archive for Rational Mechanics and Analysis, vol. 28, no. 4, pp. 265-283, 1968.

[4] F.-H. Lin and C. Liu, "Nonparabolic dissipative systems modeling the flow of liquid crystals," Communications on Pure and Applied Mathematics, vol. 48, no. 5, pp. 501-537, 1995.

[5] R. Danchin, "Density-dependent incompressible fluids in bounded domains," Journal of Mathematical Fluid Mechanics, vol. 8, no. 3, pp. 333-381, 2006.

[6] H. Kim, "A blow-up criterion for the nonhomogeneous incompressible Navier-Stokes equations," SIAM Journal on Mathematical Analysis, vol. 37, no. 5, pp. 1417-1434, 2006.

[7] J. Fan and T. Ozawa, "Regularity criteria for the 3D densitydependent Boussinesq equations," Nonlinearity, vol. 22, no. 3, pp. 553-568, 2009.

[8] X. Xu and Z. Zhang, "Global regularity and uniqueness of weak solution for the 2-D liquid crystal flows," Journal of Differential Equations, vol. 252, no. 2, pp. 1169-1181, 2012.

[9] F. Lin, J. Lin, and C. Wang, "Liquid crystal flows in two dimensions," Archive for Rational Mechanics and Analysis, vol. 197, no. 1, pp. 297-336, 2010.

[10] F.-H. Lin and C. Liu, "Partial regularity of the dynamic system modeling the flow of liquid crystals," Discrete and Continuous Dynamical Systems, vol. 2, no. 1, pp. 1-22, 1996.

[11] F.-H. Lin and C. Liu, "Existence of solutions for the EricksenLeslie system," Archive for Rational Mechanics and Analysis, vol. 154, no. 2, pp. 135-156, 2000.

[12] F. Lin and C. Liu, "Static and dynamic theories of liquid crystals," Journal of Partial Differential Equations, vol. 14, no. 4, pp. 289-330, 2001.

[13] J. Fan and B. Guo, "Regularity criterion to some liquid crystal models and the Landau-Lifshitz equations in $\mathscr{R}^{3}$," Science in China. Series A, vol. 51, no. 10, pp. 1787-1797, 2008.

[14] J. Fan and T. Ozawa, "Regularity criteria for a simplified Ericksen-Leslie system modeling the flow of liquid crystals," Discrete and Continuous Dynamical Systems. Series A, vol. 25, no. 3, pp. 859-867, 2009.

[15] Y. Zhou and J. Fan, "A regularity criterion for the nematic liquid crystal flows," Journal of Inequalities and Applications, vol. 2010, Article ID 589697, 9 pages, 2010.

[16] H. Wen and S. Ding, "Solutions of incompressible hydrodynamic flow of liquid crystals," Nonlinear Analysis: Real World Applications, vol. 12, no. 3, pp. 1510-1531, 2011.

[17] J. Fan, H. Gao, and B. Guo, "Regularity criteria for the NavierStokes-Landau-Lifshitz system," Journal of Mathematical Analysis and Applications, vol. 363, no. 1, pp. 29-37, 2010. 
[18] J. Li, "Global strong and weak solutions to nematic liquid crystal flow in two dimensions," http://arxiv.org/abs/1211.0131. 


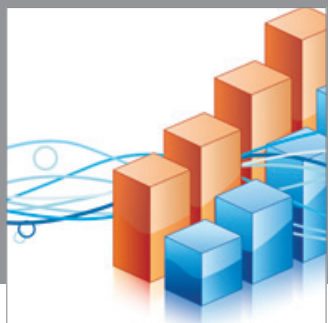

Advances in

Operations Research

mansans

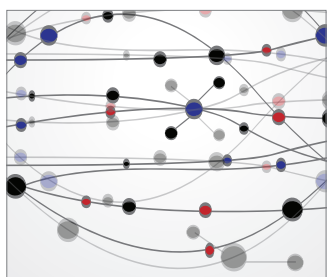

The Scientific World Journal
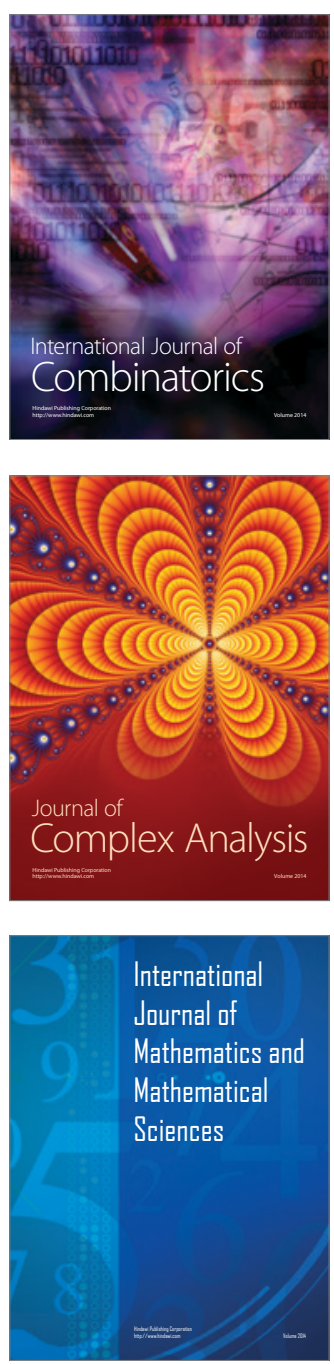
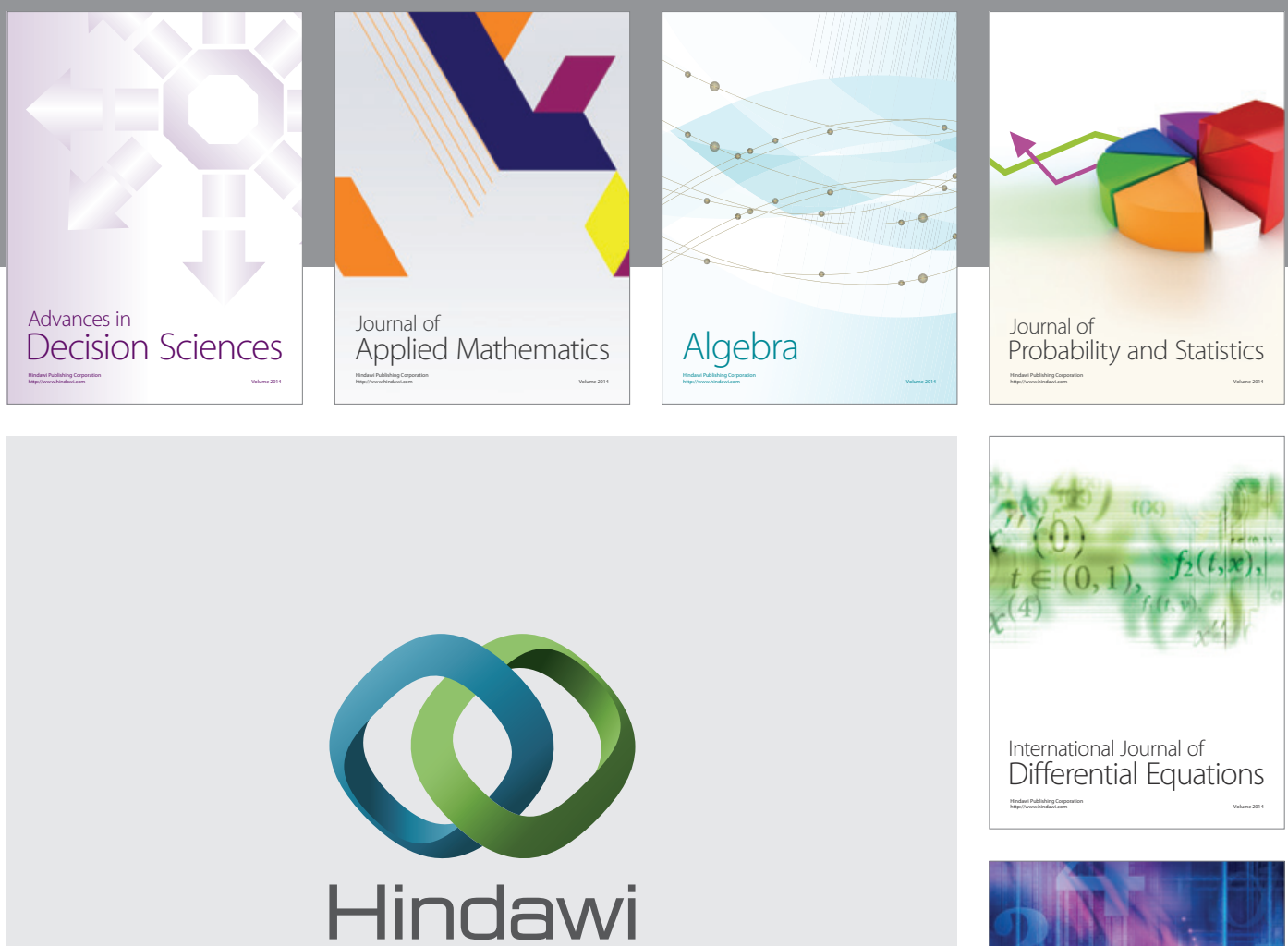

Submit your manuscripts at http://www.hindawi.com
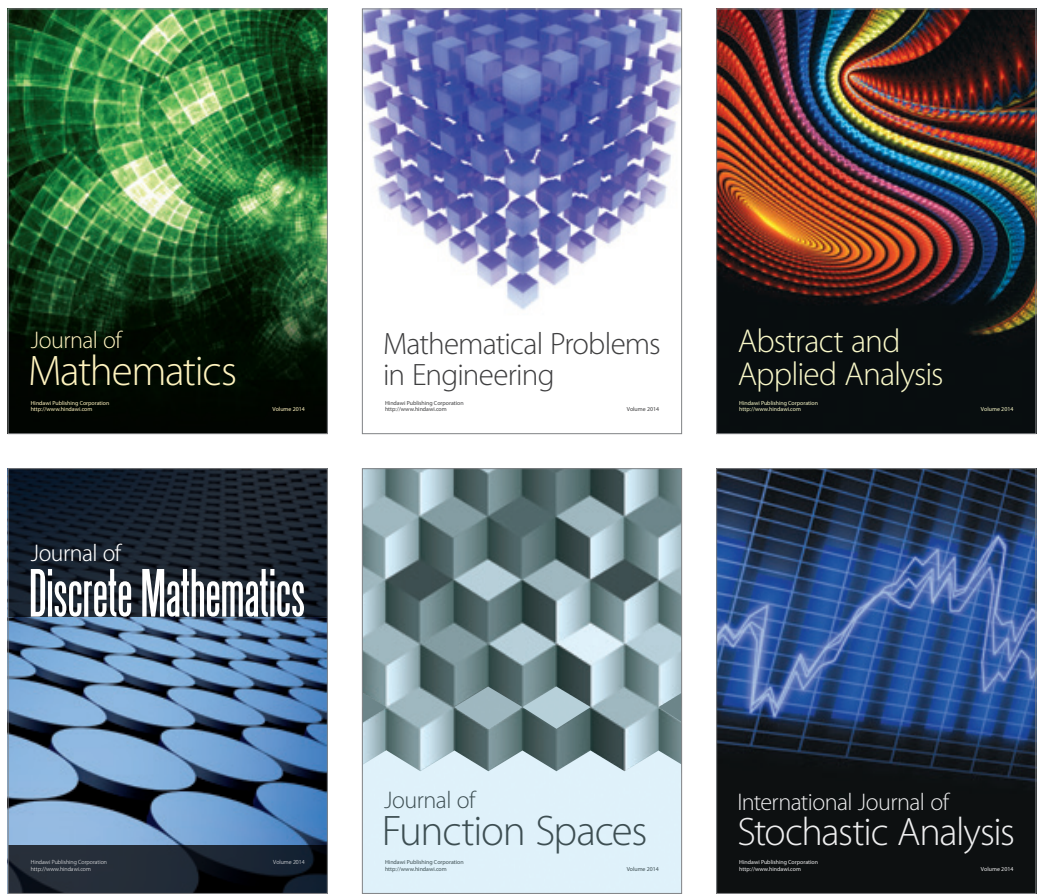

Journal of

Function Spaces

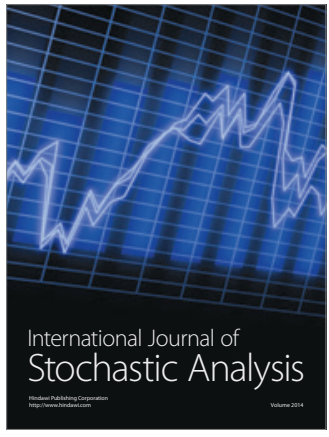

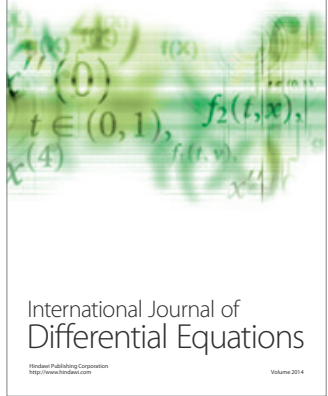
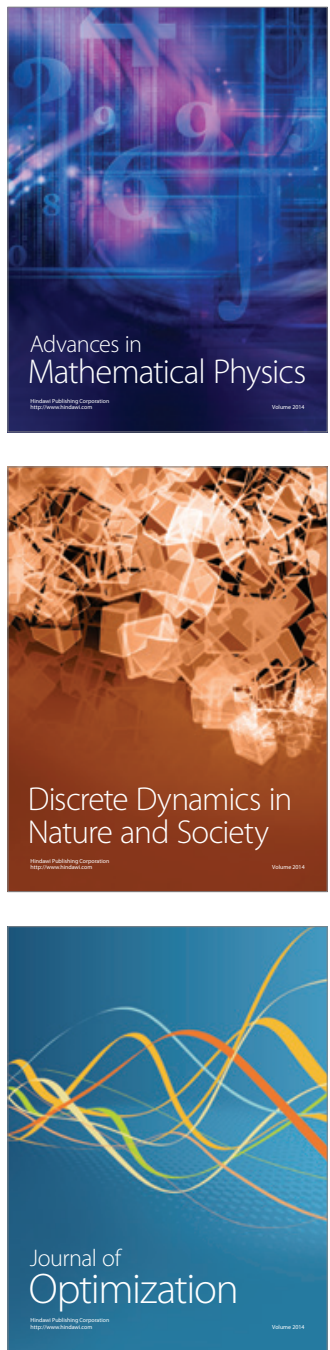